\title{
Surgical Risks and Perioperative Complications of Instrumented Lumbar Surgery in Patients with Liver Cirrhosis
}

\author{
Tung-Yi Lin, Jen-Chung Liao, Wen-Jer Chen, Lih-Huei Chen, Chi-Chien Niu, Tsai-Sheng Fu, \\ Po-Liang Lai, Tsung-Ting Tsai
}

Background: Patients with liver cirrhosis have high surgical risks due to malnutrition, impaired immunity, coagulopathy, and encephalopathy. However, there is no information in English literature about the results of liver cirrhotic patients who underwent instrumented lumbar surgery. The purpose of this study is to report the perioperative complications, clinical outcomes and determine the surgical risk factors in cirrhotic patients.

Methods: We retrospectively reviewed 29 patients with liver cirrhosis who underwent instrumented lumbar surgery between 1997 and 2009. The hepatic functional reserves of the patients were recorded according to the Child-Turcotte-Pugh scoring system. Besides, fourteen other variables and perioperative complications were also collected. To determine the risks, we divided the patients into two groups according to whether or not perioperative complications developed.

Results: Of the 29 patients, $22(76 \%)$ belonged to Child class A and 7 (24\%) belonged to Child class B. Twelve patients developed one or more complications. Patients with Child class B carried a significantly higher incidence of complications than
At a Glance Commentary
Scientific background of the subject
Malnutrition, impaired immunity, coagulopathy, and encephalopathy often develop in patients with liver cirrhosis, and this can lead to high surgical risks. However, there is no information in English literature about the results of liver cirrhotic patients underwent instrumented lumbar surgery.

\section{What this study adds to the field}
Patients with liver cirrhosis who have undergone instrumented lumbar surgery carry a high risk of developing periopera- tive complications $(41.4 \%)$, especially in those with a Child-Turcotte-Pugh score of 6 or more. A low level of albumin was also a significant risk factor. those with Child class A $(p=0.011)$. In the Child class A group, patients with 6 points had a significantly higher incidence of complications than those with 5 points $(p=0.025)$. A low level of albumin was significantly associated with higher risk, and a similar trend was also noted for the presence of ascites although statistical difference was not reached.

Conclusion: The study concludes that patients with liver cirrhosis who have undergone instrumented lumbar surgery carry a high risk of developing perioperative complications, especially in those with a Child-Turcotte-Pugh score of 6 or more.

(Biomed J 2014;37:18-23)

\section{Key words: child-turcotte-pugh score, instrumented lumbar surgery, liver cirrhosis, perioperative complications and morbidity}

$\mathrm{L}$ iver cirrhosis, the late stage of progressive hepatic fibrosis, represents an irreversible, diffuse fibrosis and nodule formation after hepatic necrosis. Patients with liver cirrhosis can have malnutrition, anemia, impaired immunity, coagulopathy, and encephalopathy. ${ }^{[1,2]}$ As a result, cirrhotic patients carry high surgical risks, and this can lead to a high perioperative complication rate, including death. ${ }^{[3-5]}$

From the Department of Orthopedics, Chang Gung Memorial Hospital at Linkou, Chang Gung University College of Medicine, Taoyuan, Taiwan

Received: Nov. 14, 2012; Accepted: Feb. 07, 2013

Correspondence to: Dr. Jen-Chung Liao, Department of Orthopedics, Chang Gung Memorial Hospital at Linkou. 5, Fusing St., Gueishan, Taoyuan 333, Taiwan (R.O.C.). Tel: 886-3-3281200 ext: 2423 Fax: 886-3-3289582; E-mail: jcl1265@adm.cgmh.org.tw

DOI: $10.4103 / 2319-4170.113376$ 
Chronic viral infection, especially chronic hepatitis B virus (HBV), hepatitis $\mathrm{C}$ virus (HCV), and alcohol consumption are the major etiologies leading to liver cirrhosis. ${ }^{[6,7]}$ In Taiwan, chronic liver disease and liver cirrhosis remains a major challenging issue due to the high prevalence of HBV of up to $15-20 \%$. ${ }^{[8,9]}$ With improved modern medical care and increased life span, spinal surgeons may increasingly encounter patients with spinal problems who have liver cirrhosis as a co-morbidity. Preoperative evaluation and management of these patients may play an important role in achieving satisfactory postoperative outcomes. The Child-Turcotte-Pugh score has been used as a factor to predict morbidity and mortality in patients with chronic liver disease ${ }^{[10]}$ For cirrhotic patients, those who are classified as Child class A have a more stable condition with lower complication rates after several different kinds of operations, including orthopedic surgery. ${ }^{[11-15]}$

To the best of our knowledge, there is no literature on the outcome of cirrhotic patients who have undergone instrumented lumbar surgery. Therefore, we retrospectively studied 29 patients with liver cirrhosis who received elective instrumented lumbar spine surgery. The purpose of this study is to report the perioperative complications, clinical outcomes and determine the surgical risk factors in cirrhotic patients.

\section{METHODS}

After obtaining IRB approval, we retrospectively reviewed patients who had undergone instrumented lumbar surgery between 1997 and 2009 in our hospital. Patients diagnosed with liver cirrhosis before surgery were included in this study. The hepatic functional reserve of the patients was recorded using the Child-Turcotte-Pugh scoring system [Table 1]. After excluding patients who had undergone instrumented spinal surgery for indications such as trauma, metastasis, and infection, a total of 29 elective cases were studied. The preoperative diagnosis for spinal surgery included degenerative spondylolisthesis, degenerative disc disease, spinal stenosis syndrome, and adjacent problems amongst others. The surgical methods included anterior interbody fusion with plate or pedicle screws, posterior decompression, pedicle screw instrumentation, discectomy, and posterior lumbar interbody fusion.

The causes of liver cirrhosis in this group of patients included chronic viral hepatitis for 24 patients (HBV in 12,

Table 1: Patients distribution in the study: Using child-turcottepugh class and score

\begin{tabular}{lcccccccr}
\hline & \multicolumn{3}{c}{ Child class } & & \multicolumn{4}{c}{ Child score } \\
\cline { 2 - 4 } \cline { 6 - 8 } & $\mathrm{A}$ & $\mathrm{B}$ & $\mathrm{C}$ & & 5 & 6 & 7 & 9 \\
\hline Complicated cases & 6 & 6 & 0 & & 4 & 5 & 1 \\
Uncomplicated cases & 16 & 1 & 0 & & 14 & 2 & 1 & 0 \\
\hline
\end{tabular}

$\mathrm{HCV}$ in 9, and both in 3), alcoholism for 4 patients, and a combination of chronic viral hepatitis and alcoholism for 1 patient. Liver cirrhosis was primarily diagnosed using the gold standard of liver biopsy with tissue proof. However, if the biopsy had not been performed, other diagnostic methods, including liver ultrasonography, abdominal CT, and a past history of liver disease and abnormal liver function would have confirmed the final diagnosis of liver cirrhosis.

Besides the Child-Turcotte-Pugh scoring system, fourteen other variables were also recorded; these included age, gender, hemoglobin, white blood cell count, platelet count, BUN level, creatinine, total bilirubin, albumin, prothrombin time/international normalized ratio (INR), cause of liver cirrhosis, presence of ascites and encephalopathy, operation time, and estimated blood loss. We documented every complication during their admission. Any reoperations, requirement of intensive care, prolonged hospital stay (over 14 days), or admission after discharge within 30 days of surgery were also defined as perioperative complications.

The clinical outcomes were evaluated according to a 5-grade patient-centered general outcome assessment questionnaire that was routinely provided to all patients undergoing elective spinal surgery at our institution. The patients in this study were interviewed by telephone after a minimal follow-up period of two years. Assessment was conducted using a 5-grade system using the following terms: "feel excellent", "feel better", "no change", "feel worse", and "feel terrible". Excellent and better were defined as successful results. Depending on whether or not perioperative complications developed, these patients were divided into two groups.

\section{Statistical analysis}

Mann-Whitney U test was used for numerical data of the two sample groups assuming equal variances. Fisher's exact test was used for categorical variables. The numerical data was recorded as mean \pm standard deviation (SD). Statistical significance was defined as $p<0.05$.

\section{RESULTS}

Of the 29 patients, 14 were male and 15 were female with a mean age of $61.38 \pm 9.35$ years $(40-78$ years). Twenty-two patients (76\%) belonged to Child class A, and 7 patients (24\%) were Child class B. There were no Child class $\mathrm{C}$ cases in this study. Twelve of the 29 patients developed one or more complications [Table 2]. The leading complication was advanced encephalopathy in 3 patients. One of these patients expired during ICU care with complications of pneumonia, encephalopathy, and acute renal failure. In the complication group, there were 6 cases of Child class A and 6 of Child class B. In the group with no complications, there 
Table 2: Patients with complications as seen in the study

\begin{tabular}{llll}
\hline Diagnosis & Etiology & Surgical method & Complication \\
\hline L1 fracture with kyphosis & HBV & T12-L2 ALIF+plating & Encephalopathy, \\
L4-5 DS & HBV & L4-5 pedicle screws+laminectomy & Prolonged stay \\
L2-3 instability L4-5 DS & HBV & L2-5 pedicle screws+laminectomy & Acute glaucoma, \\
L4-5 DS* & HBV & L4-S1 pedicle screws+L4-5 laminectomy & Pneumonia, ARF, encephalopathy \\
s/p L2-3-4 $\rightarrow$ L4-5 ADS & HCV & L2-5 pedicle screws+laminectomy & Wound infection \\
L4-5 DS & HCV & L4-5 pedicle screws+laminectomy & Wound infection \\
T12-L1 HIVD & Alcohol & T12-L1 pedicle screws+laminectomy+discectomy & Upper GI bleeding \\
L4-5 HIVD & Alcohol, HBV & L4-5 pedicle screws+laminectomy+discectomy & Implant loosening \\
L1-2 Schwannoma, L4-5 DS & HBV, HCV & L4-5 pedicle screws+laminectomy+excision tumor & Ileus, encephalopathy \\
L4-5, L5-S1 DS & HBV, HCV & L4-S1 pedicle screws+L4-5 laminectomy+L4-5 and L5-S1 PLIF & Prolonged stay \\
L3-4, L4-5 DS & HCV & L3-5 pedicle screws+laminectomy & Ascites \\
L2-3, L4-5 DS & HBV & L2-5 pedicle screws+laminectomy & Acute glaucoma
\end{tabular}

Abbreviations: DS: Degenerative spondylolisthesis; ASD: Adjacent segmental degeneration; ALIF: Anterior lumbar interbody fusion; PLIF: Posterior lumbar interbody fusion; ARF: Acute renal failure; HBV: Hepatitis-B viral infection; HCV: Hepatitis-C viral infection; HIVD: Herniated intervertebral disc; *: The fourth case had expired

were 16 cases of Child A class and only one of Child B class. A significantly higher incidence of complications occurred in patients with Child class B (6 out of $7(85.7 \%))$ than in those with Child class A (6 out of $22(27.3 \% ; p=0.011)$ ). In the group with Child class A, patients with a score of 6 points had a significantly higher incidence of complications (66.7\%) than those with 5 points $(12.5 \% ; p=0.025)$.

To determine the risk factors for complications in cirrhotic patients, the Child system and another 14 variables associated with liver disease and surgery were recorded [Table 3]. A low level of albumin demonstrated a significant difference $(p=0.002)$ between the complication and no-complication group, and a similar trend was also noted for the presence of ascites although the statistical difference was not reached $(p=0.06)$. No significant difference was found between the 2 groups in terms of age, hemoglobin, white cell count, platelet count, prothrombin time, INR, renal function (BUN/creatinine), level of bilirubin, operating time, and cause of liver cirrhosis. Duration of hospital stay was greater in the complication group (mean $>20$ days) than in the group with no complications ( $p=0.007$ ).

Our outcome assessment questionnaire revealed that in the no-complication group, a successful result was reported by $82 \%(14 / 17)$ of patients, and this significantly decreased to only $50 \%(6 / 12)$ in the complications group $(p=0.064)$.

\section{DISCUSSION}

The multiple functions of the liver include synthesis of bioactive molecules, metabolism, detoxification, and filtering of blood from the portal venous system. Liver cirrhosis, the end stage of liver fibrosis, may lead to an immunocompromised state, bleeding tendency, portal hypertension, encephalopathy, and multi-organ complications, such as hepatorenal and hepatopulmonary syndrome. ${ }^{[16]}$ The opera- tive risk is related to liver decompensation, associated morbidity, and the type of surgery. The hemodynamic changes during anesthesia and surgery, and also perioperative factors such as sepsis and blood loss may cause poor results in a cirrhotic patient. ${ }^{[1,5]}$ The Child-Turcotte-Pugh system includes the albumin level, serum bilirubin, prolonged prothrombin time, ascites condition, and the severity of encephalopathy. This system reflects the severity of the liver condition on the basis of metabolic-synthetic functions, the degree of portal hypertension and represents the predicting risk factor for perioperative complications. ${ }^{[3,14,17,18]}$

Advanced liver disease is related to high complication and mortality rates in abdominal surgery and also after various other kinds of operations. Suman et al., studied 44 patients with liver cirrhosis undergoing cardiac surgery. The results displayed that patients with Child-Pugh scores of $\leq 7$ (Child A) had better outcomes after surgery with much less hepatic decompensation and death. ${ }^{[12]}$ Hsieh et al., studied the outcome of cirrhotic patients who had undergone hip arthroplasty, and results showed high perioperative complication rates of up to $26.7 \% .{ }^{[13]}$ Predicting risk factors in this study were similar to those in our study, including decreased albumin level, ascites, and increased operative blood loss.

The clinical outcome of lumbar surgery differs with the operative procedure, patient condition, and complications. ${ }^{[19]}$ The perioperative complication rates of instrumented lumbar surgery showed variable results between different studies. ${ }^{[20-24]}$ Sharpiro et al., reported a series of 299 cases of spinal instrumented surgery with a low overall complication rate $10 / 299(3 \%)$, a reoperative rate of $2 \%$, and one perioperative death unrelated to instrumentation. ${ }^{[22]}$ Ghogawala et al., treated lumbar stenosis and low-grade degenerative spondylolisthesis resulting in high fusion rates (93\%), and low complication rates with one perioperative pneumonia 
Table 3: Patient demographic data (complicated vs. uncomplicated group) as seen in the study

\begin{tabular}{lccc}
\hline & $\begin{array}{c}\text { Complicated } \\
\text { group } \\
(n=12)\end{array}$ & $\begin{array}{c}\text { Uncomplicated } \\
\text { group } \\
(n=17)\end{array}$ & $p$ value \\
\hline Age (years) & $63.17 \pm 7.52$ & $60.12 \pm 10.49$ & 0.394 \\
Sex (F/M) & $9 / 3$ & $6 / 11$ & 0.060 \\
Child score (points) & $6.50 \pm 1.09$ & $5.24 \pm 0.56$ & $0.001 *$ \\
Hemoglobin (g/dL) & $10.86 \pm 2.20$ & $12.52 \pm 2.80$ & 0.073 \\
WBC (1000 cells/mm $)$ & $5.28 \pm 1.75$ & $6.64 \pm 1.94$ & 0.053 \\
Platelet (1000 cells/mm sm $\left.^{3}\right)$ & $112.37 \pm 54.22$ & $133.00 \pm 81.27$ & 0.471 \\
PT (seconds) & $12.31 \pm 4.62$ & $12.59 \pm 2.23$ & 0.845 \\
INR & $1.26 \pm 0.36$ & $1.14 \pm 0.19$ & 0.616 \\
BUN (mg/dL) & $16.08 \pm 5.68$ & $21.47 \pm 13.22$ & 0.419 \\
Creatinine (mg/dL) & $1.14 \pm 0.89$ & $1.72 \pm 1.96$ & 0.073 \\
T-Bilirubin (mg/dL) & $1.23 \pm 0.84$ & $1.06 \pm 0.55$ & 0.744 \\
Albumin (mg/dL) & $3.37 \pm 0.45$ & $4.04 \pm 0.56$ & $0.002 *$ \\
Ascites (Y/N) & $3 / 9$ & $0 / 17$ & 0.060 \\
Encephalopathy (Y/N) & $1 / 11$ & $0 / 17$ & 0.414 \\
Operation time (minutes) & $223.33 \pm 130.27$ & $210.76 \pm 58.30$ & 0.556 \\
Blood loss (mL) & $1287.50 \pm 708.75$ & $826.47 \pm 525.04$ & 0.088 \\
Etiology of liver cirrhosis & & & \\
Alcohol & 2 & 3 & \\
HBV & 5 & 7 & 0.793 \\
HCV & 3 & 6 & \\
HBV+HCV & 2 & 1 & \\
Hospit say (days) & $20.58 \pm 17.69$ & $7.94 \pm 1.75$ & $<0.001 *$
\end{tabular}

Hospital stay (days) $\quad 20.58 \pm 17.69 \quad 7.94 \pm 1.75<0.001 *$

Abbreviations: F: Female; M: Male; WBC: White blood cell count; PT: Prothrombin time; INR: International normalized ratio; BUN: Blood urea nitrogen; T-bilirubin: Total bilirubin; Y: Yes; N: No; HBV: Hepatitis-B viral infection; HCV: Hepatitis-C viral infection;

*: Significant difference

and one wound infection of 14 instrumented cases. ${ }^{[23]}$ Booth et al., reported instrumented fusion with decompression for degenerative spondylolisthesis, resulting in a high satisfaction rate and a low rate $(2 \%)$ of major complications. ${ }^{[24]}$ The reported complication rates in past literature provide a comparison between the general population and cirrhotic patients in this study. In our study, patients with cirrhosis demonstrated high perioperative complications rates up to $41.4 \%$ (12/29). Two cases in our series developed acute glaucoma after spinal surgery. Acute glaucoma or even visual loss has been reported as a complication after spinal surgery in the literature, ${ }^{[25,26]}$ which might be related to prolonged prone positioning resulting from cirrhosis-related bleeding tendency. The two patients had unsatisfactory recovery due to prolonged post-op recovery time (prolonged stay over 14 days). In addition, one patient had postoperative refractory leg pain with conservative treatment failure requiring revision surgery. Pedicle screw loosening was noticed during the $2^{\text {nd }}$ operation, so we recorded the patient's outcome as complicated with implant loosening.

Advancements in medical care and improvement in instrumentations may lead to higher demands by patients for elective spinal surgery and expectations of better results. However, patients may have major medical underlying illnesses that lead to increased surgical risks. In Taiwan, there is a high prevalence of chronic viral hepatitis that results in liver cirrhosis and hepatoma. ${ }^{[27]}$ Thus, patients with liver cirrhosis who undergo instrumented lumbar spinal surgery represent a common and big challenge for spinal surgeons. ${ }^{[28]}$ In our study, patients with Child A class status had a satisfactory results and low complication rates, especially when compared with Child B class patients. A previous study by Shih et al., that investigated total knee replacements in cirrhotic patients, and reported similar results. ${ }^{[15]}$ Surgeons may consider performing elective instrumented spinal surgery for this class of patients. They may choose to either provide pre-operative hepatic status correction, or consult the patient cautiously on the risk of complications for Child B class patients. Perioperative complications often result in unsatisfactory clinical outcomes and poor postoperative recovery. The surgeon should educate these patients on the possibility of development of early complications during the preoperative planning period.

In the present series, ascites status and albumin level were considered as risk factors between the group with complications and the group with no complications. These results support that preoperative treatment and correction could benefit those with ascites and low albumin. Methods used in our institution include the use of diuretics and abdominal tapping to control the ascites. Albumin supplement could also be given to correct hypoalbuminemia. Besides, platelet and plasma transfusions should be performed more aggressively to decrease the intraoperative blood loss. From our experience, the rate of perioperative complications and mortality decreased, and patients were more satisfied with the clinical outcome. In addition to correction of abnormal biochemical data, the bleeding tendency, prolonged operative time, and high perioperative complication rates should be well explained to cirrhotic patients. Some of the patients were not fully satisfied, but could accept the results due to pre-operative understanding of possible complications resulting from their underlying disease. The only patient who expired had cirrhosis due to HBV infection. He had undergone implantation of pedicular screws from L4 to S1 and a laminectomy at the L4 to L5 level under the degenerative stenosis at L4-L5. Postoperative ICU care was required because of acute renal failure and acute onset of encephalopathy. $\mathrm{He}$ developed pneumonia and a high fever, and then expired after developing multi-organ failure. Unlike operations for trauma, tumor, or infection, more satisfactory clinical outcomes are expected for elective surgery. A full preoperative survey and managements to improve the condition of cirrhotic patients may be needed, especially in controlling ascites with medications and albumin supplementation. 
There were some limitations in this study. One is the relatively small case numbers, due to our focus on elective instrumented lumber surgery and exclusion of patients with infection and metastasis. There may also be bias due to differing diagnosis and differing surgical methods. Another issue is the lack of a matched-case control group. However, many review studies have demonstrated more satisfactory results from treating patients without liver cirrhosis as mentioned above in the discussion. No patients with Child class $\mathrm{C}$ were included in this study, as these patients may have a predictably higher rate of mortality and are at extremely high risk for anesthesia. With our results, it can be expected that the morbidity and mortality of Child class $\mathrm{C}$ patients will be much higher, rendering this population of patients unsuitable for elective surgery. Finally, more follow-up data is required to determine long-term results and complications.

In conclusion, this study showed that patients with liver cirrhosis who have undergone instrumented lumbar surgery may have a high risk of perioperative complications, especially in those with a Child score of 6 or higher. Low albumin and presence of ascites were considered as risk factors with complications. These perioperative complications might attribute to unsatisfactory clinical outcomes. Whenever elective instrumented lumber surgery needs to be performed for patients with liver cirrhosis, the risk factors should be evaluated before an operation, and urgent surgical interventions should be avoided for patients in poor clinical condition.

\section{REFERENCES}

1. Friedman LS. The risk of surgery in patients with liver disease. Hepatology 1999;29:1617-23.

2. Blake JC, Sprengers D, Grech P, McCormick PA, McIntyre N, Burroughs AK. Bleeding time in patients with hepatic cirrhosis. BMJ 1990;301:12-5.

3. Mansour A, Watson W, Shayani V, Pickleman J. Abdominal operations in patients with cirrhosis: Still a major surgical challenge. Surgery 1997;122:730-5.

4. Rice HE, O'Keefe GE, Helton WS, Johansen K. Morbid prognostic features in patients with chronic liver failure undergoing nonhepatic surgery. Arch Surg 1997;132:880-4.

5. Ziser A, Plevak DJ, Wiesner RH, Rakela J, Offord KP, Brown DL. Morbidity and mortality in cirrhotic patients undergoing anesthesia and surgery. Anesthesiology 1999;90:42-53.

6. Hayashi PH, Di Bisceglie AM. The progression of hepatitis B- and $\mathrm{C}$-infections to chronic liver disease and hepatocellular carcinoma: Epidemiology and pathogenesis. Med Clin North Am 2005;89:371-89.

7. Mendenhall CL, Seeff L, Diehl AM, Ghosn SJ, French SW, Gartside PS, et al. Antibodies to hepatitis B virus and hepatitis C virus in alcoholic hepatitis and cirrhosis: their prevalence and clinical relevance. The VA Cooperative Study Group (No. 119). Hepatology 1991;14:581-9.

Biomed J Vol. 37 No. 1

January - February 2014
8. Chen DS, Sung JL. Hepatitis B virus infection on Taiwan. N Engl J Med 1977; 297:668-9.

9. Stevens CE, Beasley RP, Tsui J, Lee WC. Vertical transmission of hepatitis B antigen in Taiwan. N Engl J Med 1975;292:771-4.

10. Friedman LS. Surgery in the patient with liver disease. Trans Am Clin Climatol Assoc 2010;121:192-204.

11. Telem DA, Schiano T, Goldstone R, Han DK, Buch KE, Chin EH, et al. Factors that predict outcome of abdominal operations in patients with advanced cirrhosis. Clin Gastroenterol Hepatol 2010;8:451-7.

12. Suman A, Barnes DS, Zein NN, Levinthal GN, Connor JT, Carey WD. Predicting outcome after cardiac surgery in patients with cirrhosis: A comparison of Child-Pugh and MELD scores. Clin Gastroenterol Hepatol 2004;2:719-23.

13. Hsieh PH, Chen LH, Lee MS, Chen CH, Yang WE, Shih CH. Hip arthroplasty in patients with cirrhosis of the liver. J Bone Joint Surg Br 2003;85:818-21.

14. O'Leary JG, Friedman LS. Predicting surgical risk in patients with cirrhosis: From art to science. Gastroenterology 2007;132:1609-11.

15. Shih LY, Cheng CY, Chang CH, Hsu KY, Hsu RW, Shih HN. Total knee arthroplasty in patients with liver cirrhosis. J Bone Joint Surg Am 2004;86A: 335-41.

16. Gholson CF, Provenza JM, Bacon BR. Hepatologic considerations in patients with parenchymal liver disease undergoing surgery. Am J Gastroenterol 1990;85:487-96.

17. Lu W, Wai CT. Surgery in patients with advanced liver cirrhosis: A Pandora's box. Singapore Med J 2006;47:152-5.

18. Garrison RN, Cryer HM, Howard DA, Polk HC Jr. Clarification of risk factors for abdominal operations in patients with hepatic cirrhosis. Ann Surg 1984;199:648-55.

19. Deyo RA, Cherkin DC, Loeser JD, Bigos SJ, Ciol MA. Morbidity and mortality in association with operations on the lumbar spine. The influence of age, diagnosis, and procedure. J Bone Joint Surg Am 1992;74:536-43.

20. Fritzell P, Hagg O, Nordwall A. Complications in lumbar fusion surgery for chronic low back pain: Comparison of three surgical techniques used in a prospective randomized study. A report from the Swedish Lumbar Spine Study Group. Eur Spine J 2003;12:178-89.

21. Nork SE, Hu SS, Workman KL, Glazer PA, Bradford DS. Patient outcomes after decompression and instrumented posterior spinal fusion for degenerative spondylolisthesis. Spine (Phila Pa 1976) 1999;24:561-9.

22. Shapiro SA, Snyder W. Spinal instrumentation with a low complication rate. Surg Neurol 1997;48:566-74.

23. Ghogawala Z, Benel EC, Amin-Hanjani S, Barker FG $2^{\text {nd }}$, Harrington JF, Magge SN, et al. Prospective outcomes evaluation after decompression with or without instrumented fusion for lumbar stenosis and degenerative Grade I spondylolisthesis. J Neurosurg Spine 2004;1:267-72.

24. Booth KC, Bridwell KH, Eisenberg BA, Baldus CR, Lenke LG. Minimum 5-year results of degenerative spondylolisthesis treated with decompression and instrumented posterior fusion. Spine (Phila Pa 1976) 1999;24:1721-7.

25. Singer MS, Salim S. Bilateral acute angle-closure glaucoma as a complication of facedown spine surgery. Spine J 2010;10:e7-9. 
26. Kamming D, Clarke S. Postoperative visual loss following prone spinal surgery. Br J Anaesth 2005;95:257-60.

27. Tsai MC, Kee KM, Chen YD, Lin LC, Tsai LS, Chen HH, et al. Excess mortality of hepatocellular carcinoma and morbidity of liver cirrhosis and hepatitis in HCV-endemic areas in an HBV-endemic country: Geographic variations among 502 villages in southern Taiwan. J Gastroenterol Hepatol 2007;22:92-8.

28. Jackson FC, Christophersen EB, Peternel WW, Kirimli B. Preoperative management of patients with liver disease. Surg Clin North Am 1968;48:907-30. 